PROCEEDINGS OF THE

AMERICAN MATHEMATICAL SOCIETY

Volume 126, Number 2, February 1998, Pages 441-446

S 0002-9939(98)04065- 9

\title{
UNIQUE CONTINUATION ON THE BOUNDARY FOR DINI DOMAINS
}

\author{
IGOR KUKAVICA AND KAJ NYSTRÖM
}

(Communicated by Christopher D. Sogge)

\begin{abstract}
We show that the normal derivative of a harmonic function which vanishes on an open subset of the boundary of a Dini domain cannot vanish on a subset of positive surface measure.
\end{abstract}

\section{INTRODUCTION}

In $[\mathrm{L}]$, the following unique continuation question was raised: If $u$ is a harmonic function in a Lipschitz domain $\Omega$ which vanishes on an open subset $\Gamma$ of the boundary $\partial \Omega$, and if $u$ is not identically zero, does it follow that the surface measure of the set $\{x \in \Gamma: \nabla u=0\}$ is zero? In [L], it was shown that the answer is affirmative if $\Omega$ is a $C^{1,1}$ domain. In $[\mathrm{AEK}]$, it was proven that the answer is affirmative also for convex domains. Further, in $[\mathrm{AE}]$, it was proven that the fact holds for Dini domains, and thus, in particular, for $C^{1, \alpha}$ domains for all $\alpha \in(0,1]$. The approach in $[\mathrm{AE}]$ consists of a local change of variables around points on the boundary, which transforms the harmonic operator to a more general elliptic one, while the boundary becomes (in a certain sense) locally convex with respect to this operator.

The purpose of the present paper is to present a short and elementary proof of the Adolfsson-Escauriaza result. By [AEK], it is sufficient to prove that $u$ satisfies a uniform doubling type condition on $\Gamma$. This is done by studying the logarithmic convexity of averages of $u^{2}$ over the balls centered at points $x_{0} \in \Gamma$, rather than spherical shell averages as in $[\mathrm{L}],[\mathrm{AEK}]$, and $[\mathrm{AE}]$, and take into account the fact that a Dini domain is locally star-shaped with respect to suitably chosen points close to $x_{0}$.

\section{THE MAIN RESULTS}

In this section, we recall the definition of a Dini domain and state the main results.

Definition 2.1. An open connected domain $\Omega \subseteq \mathbb{R}^{d}$ is a Dini domain if for each point $x_{0} \in \partial \Omega$, there exists a local coordinate system $\left(x^{\prime}, x_{d}\right) \in \mathbb{R}^{d-1} \times \mathbb{R}, R_{0}>0$, and a function $\phi: \mathbb{R}^{d-1} \rightarrow \mathbb{R}$ such that

(i) $B_{R_{0}}\left(x_{0}\right) \cap \Omega=\left\{\left(x^{\prime}, x_{d}\right) \in B_{R_{0}}\left(x_{0}\right): x_{d}<\phi\left(x^{\prime}\right)\right\}$,

(ii) $B_{R_{0}}\left(x_{0}\right) \cap \partial \Omega=\left\{\left(x^{\prime}, x_{d}\right) \in B_{R_{0}}\left(x_{0}\right): x_{d}=\phi\left(x^{\prime}\right)\right\}$,

Received by the editors May 13, 1996 and, in revised form, July 30, 1996.

1991 Mathematics Subject Classification. Primary 31B05.

(C)1998 American Mathematical Society 
(iii) $\left|\nabla \phi\left(x_{1}^{\prime}\right)-\nabla \phi\left(x_{2}^{\prime}\right)\right| \leq \psi\left(\left|x_{1}^{\prime}-x_{2}^{\prime}\right|\right)$ for all $\left(x_{1}^{\prime}, \phi\left(x_{1}^{\prime}\right)\right),\left(x_{2}^{\prime}, \phi\left(x_{2}^{\prime}\right)\right) \in B_{R_{0}}\left(x_{0}\right)$ where $\psi$ satisfies

$$
\int_{0}^{1} \frac{\psi(r)}{r} d r<\infty
$$

Theorem 2.2. Let $\Omega$ be a Dini domain, and let $\Gamma$ be an open subset of the boundary $\partial \Omega$. If $u \in C(\Gamma \cup \Omega)$ is harmonic in $\Omega$, and if $u$ vanishes on $\Gamma$, then, for every $x_{0} \in \Gamma$

$$
\sup _{x_{0} \in \Gamma} \sup _{r \in(0,1)} \frac{\int_{B_{2 r}\left(x_{0}\right) \cap \Omega} u^{2} d x}{\int_{B_{r}\left(x_{0}\right) \cap \Omega} u^{2} d x}<\infty .
$$

The following corollary of Theorem 2.2 is our main result.

Theorem 2.3. Let $\Omega$ be a Dini domain, and let $\Gamma$ be an open subset of the boundary $\partial \Omega$. If $u \in C(\Gamma \cup \Omega)$ is harmonic in $\Omega$, and if $u$ vanishes on $\Gamma$, then the surface measure of $\{x \in \Gamma:|\nabla u(x)|=0\}$ is zero.

Proof of Theorem 2.3. It is proven in [AEK] (or cf. [L]) that Theorem 2.2 implies Theorem 2.3.

Theorem 2.2 is proven in the next section.

\section{Proof of Theorem 2.2}

Let $\Omega \subseteq \mathbb{R}^{d}$ be a Lipschitz domain, and let $u$ be harmonic in $\Omega$.

For any $x_{0} \in \bar{\Omega}$, denote

$$
H_{x_{0}}(r)=\int_{B_{r}\left(x_{0}\right) \cap \Omega} u^{2} d x .
$$

Lemma 3.1. Let $B_{R_{0}}\left(x_{0}\right) \cap \Omega$ be star-shaped with respect to some $x_{0} \in \bar{\Omega}$, and assume that $u$ vanishes continuously on $B_{R_{0}}\left(x_{0}\right) \cap \partial \Omega$. If $0<r_{1}<r_{2}<r_{3}<R_{0}$, then

$$
\frac{\log \frac{H_{x_{0}}\left(r_{2}\right)}{H_{x_{0}}\left(r_{1}\right)}}{\log \frac{r_{2}}{r_{1}}} \leq \frac{\log \frac{H_{x_{0}}\left(r_{3}\right)}{H_{x_{0}}\left(r_{2}\right)}}{\log \frac{r_{3}}{r_{2}}} .
$$

The interior version of the above lemma was obtained in $[\mathrm{K}]$.

Proof of Lemma 3.1. Assume $x_{0}=0$; also, denote $H(r)=H_{0}(r)$ and $B_{r}=B_{r}(0)$. Fix an arbitrary $r \in\left(0, R_{0}\right)$. Since $B_{R_{0}} \cap \Omega$ is star-shaped with respect to 0 , and since $u$ vanishes on $B_{R} \cap \partial \Omega$,

$$
\begin{aligned}
H^{\prime}(r) & =\int_{\left(\partial B_{r}\right) \cap \Omega} u^{2} d \sigma=\int_{\partial\left(B_{r} \cap \Omega\right)} u^{2} d \sigma=\frac{1}{r} \int_{B_{r} \cap \Omega} \operatorname{div}\left(u^{2} x\right) d x \\
& =\frac{d}{r} H(r)+\frac{2}{r} \int_{B_{r} \cap \Omega} u x \cdot \nabla u d x .
\end{aligned}
$$

Denote

$$
\begin{aligned}
I(r) & =2 \int_{B_{r} \cap \Omega} u x \cdot \nabla u d x=-\int_{B_{r} \cap \Omega} u \nabla u \cdot \nabla\left(r^{2}-|x|^{2}\right) d x \\
& =\int_{B_{r} \cap \Omega}|\nabla u|^{2}\left(r^{2}-|x|^{2}\right) d x
\end{aligned}
$$


where we again used that $u$ vanishes on $B_{R_{0}} \cap \partial \Omega$ and that $u$ is harmonic. Differentiating the last expression, we get

$$
I^{\prime}(r)=2 r \int_{B_{r} \cap \Omega}|\nabla u|^{2} d x+\int_{\left(\partial B_{r}\right) \cap \Omega}|\nabla u|^{2}\left(r^{2}-|x|^{2}\right) d \sigma=2 r \int_{B_{r} \cap \Omega}|\nabla u|^{2} d x .
$$

Note that, using integration by parts,

$$
\begin{aligned}
\frac{1}{r} \int_{B_{r} \cap \Omega} x & \cdot \nabla\left(|\nabla u|^{2}\right)\left(r^{2}-|x|^{2}\right) d x \\
= & -\frac{d}{r} I(r)+\frac{2}{r} \int_{B_{r} \cap \Omega}|x|^{2}|\nabla u|^{2} d x+\frac{1}{r} \int_{B_{r} \cap \partial \Omega}|\nabla u|^{2}\left(r^{2}-|x|^{2}\right) x \cdot n d \sigma \\
= & -\frac{d+2}{r} I(r)+\frac{2}{r} \int_{B_{r} \cap \Omega}|\nabla u|^{2}\left(r^{2}-|x|^{2}\right) d x \\
& \quad+\frac{2}{r} \int_{B_{r} \cap \Omega}|x|^{2}|\nabla u|^{2} d x+\frac{1}{r} \int_{B_{r} \cap \partial \Omega}|\nabla u|^{2}\left(r^{2}-|x|^{2}\right) x \cdot n d \sigma \\
= & -\frac{d+2}{r} I(r)+2 r \int_{B_{r} \cap \Omega}|\nabla u|^{2} d x+\frac{1}{r} \int_{B_{r} \cap \partial \Omega}|\nabla u|^{2}\left(r^{2}-|x|^{2}\right) x \cdot n d \sigma,
\end{aligned}
$$

where $n$ denotes the outward unit normal on $\partial \Omega$. Therefore,

$$
\begin{aligned}
I^{\prime}(r)= & \frac{d+2}{r} I(r)+\frac{2}{r} \int_{B_{r} \cap \Omega} x_{k}\left(\partial_{k} \partial_{j} u\right)\left(\partial_{j} u\right)\left(r^{2}-|x|^{2}\right) d x \\
& -\frac{1}{r} \int_{B_{r} \cap \partial \Omega}|\nabla u|^{2}\left(r^{2}-|x|^{2}\right) x \cdot n d \sigma,
\end{aligned}
$$

whence, integrating by parts (with respect to $j$ ) the second expression on the righthand side,

$$
\begin{aligned}
I^{\prime}(r)=\frac{d+2}{r} I(r) & -\frac{2}{r} \int_{B_{r} \cap \Omega}|\nabla u|^{2}\left(r^{2}-|x|^{2}\right) d x+\frac{4}{r} \int_{B_{r} \cap \Omega}(x \cdot \nabla u)^{2} d x \\
& +\frac{2}{r} \int_{B_{r} \cap \partial \Omega}(x \cdot \nabla u)(n \cdot \nabla u)\left(r^{2}-|x|^{2}\right) d \sigma \\
& -\frac{1}{r} \int_{B_{r} \cap \partial \Omega}|\nabla u|^{2}\left(r^{2}-|x|^{2}\right) x \cdot n d \sigma .
\end{aligned}
$$

The sum of the last two terms is nonnegative since on $B_{R} \cap \partial \Omega$ the tangential derivative of $u$ vanishes and $x \cdot n \geq 0$ almost everywhere on $B_{r} \cap \partial \Omega$. We get

$$
I^{\prime}(r) \geq \frac{d}{r} I(r)+\frac{4}{r} \int_{B_{r} \cap \Omega}(x \cdot \nabla u)^{2} d x .
$$

Denoting by

$$
N(r)=\frac{I(r)}{H(r)}
$$


the frequency function, we obtain by differentiation

$$
\begin{aligned}
N^{\prime}(r) & =\frac{1}{H(r)^{2}}\left(I^{\prime}(r) H(r)-I(r) H^{\prime}(r)\right) \\
& \geq \frac{4}{r H(r)^{2}}\left(\int_{B_{r} \cap \Omega}(x \cdot \nabla u)^{2} d x \int_{B_{r} \cap \Omega} u^{2} d x-\left(\int_{B_{r} \cap \Omega} u x \cdot \nabla u d x\right)^{2}\right) \geq 0,
\end{aligned}
$$

and we conclude that $N(r)$ is an increasing function of $r \in\left(0, R_{0}\right)$. Now, (3.1) implies

$$
\log \frac{H\left(r_{2}\right)}{H\left(r_{1}\right)}=d \log \frac{r_{2}}{r_{1}}+\int_{r_{1}}^{r_{2}} \frac{N(r)}{r} d r \leq\left(d+N\left(r_{2}\right)\right) \log \frac{r_{2}}{r_{1}} .
$$

Similarly,

$$
\log \frac{H\left(r_{3}\right)}{H\left(r_{2}\right)}=d \log \frac{r_{3}}{r_{2}}+\int_{r_{2}}^{r_{3}} \frac{N(r)}{r} d r \geq\left(d+N\left(r_{2}\right)\right) \log \frac{r_{3}}{r_{2}} .
$$

The asserted inequality is then obtained by combining (3.2) and (3.3).

Consider the following setting. Let $\phi: \mathbb{R}^{d-1} \rightarrow \mathbb{R}$ be continuously differentiable. Denote

$$
\Omega=B_{2} \cap\left\{\left(x^{\prime}, x_{d}\right) \in \mathbb{R}^{d-1} \times \mathbb{R}: \phi\left(x^{\prime}\right)<x_{d}\right\},
$$

where $B_{2}=B_{2}(0)$. For every $r \in(0,2)$, let

$$
\Lambda(r)=\sup \left|n\left(x_{2}\right)-n\left(x_{1}\right)\right|
$$

where the supremum is taken over all $x_{1}, x_{2} \in B_{2} \cap \partial \Omega$ such that $\left|x_{2}-x_{1}\right| \leq r$, and where $n(x)$ denotes the outward unit normal at $x \in B_{2} \cap \partial \Omega$. Note that $\Lambda$ is non-decreasing. Assume also $\phi(0)=0$ (i.e., $0 \in \partial \Omega$ ).

Throughout this section, we assume that $u \in C(\bar{\Omega})$ is harmonic in $\Omega$ and that it vanishes on $B_{2} \cap \partial \Omega$. Theorem 2.2 will be an immediate consequence of the following lemma.

Lemma 3.2. Assume

$$
\int_{0}^{2} \frac{\Lambda(r)}{r} d r<\infty
$$

$\Lambda(r) \neq 0$ for $r \in(0,2)$, and $\Lambda(2) \leq 1 / 32$. Then

$$
C_{1}=\prod_{m=1}^{\infty} \frac{\log \frac{2+16 \Lambda\left(2^{-m}\right)}{1-16 \Lambda\left(2^{-m}\right)}}{\log \frac{4-16 \Lambda\left(2^{-m}\right)}{2+16 \Lambda\left(2^{-m}\right)}}<\infty,
$$

and for all $x_{0} \in B_{1} \cap \partial \Omega$, we have

$$
H_{x_{0}}(2 r) \leq C_{2} H_{x_{0}}(r), \quad r \in\left(0, \frac{1}{2}\right),
$$

where $C_{2}=\left(H_{x_{0}}(1) / H_{x_{0}}(1 / 2)\right)^{2 C_{1}}$. 
Proof of Lemma 3.2. Note that the function

$$
f(x)=\frac{\log \frac{2+x}{1-x}}{\log \frac{4-x}{2+x}}
$$

is increasing and continuously differentiable on $(-2,1)$. Since also $f(0)=1$, we have

$$
1 \leq f(x) \leq 1+e^{C x}, \quad x \in[0,1 / 2],
$$

for some constant $C>0$. Hence, the product in (3.5) converges provided

$$
\sum_{m=1}^{\infty} \Lambda\left(2^{-m}\right)<\infty
$$

However, the last fact follows directly from (3.4) taking into account that $\Lambda$ is non-decreasing.

Without loss of generality, we may assume $x_{0}=0$ and $n(0)=(0,1)$.

Fix any $r \in(0,1)$. Let $\left(x_{1}^{\prime}, \phi\left(x_{1}^{\prime}\right)\right),\left(x_{2}^{\prime}, \phi\left(x_{2}^{\prime}\right)\right) \in B_{1}$ be arbitrary points. It is easy to check that $\left|\nabla \phi\left(x_{j}^{\prime}\right)\right| \leq 3 \Lambda(r) / 2$ for $j=1,2$; the mean value theorem implies

$$
\left|\phi\left(x_{2}^{\prime}\right)-\phi\left(x_{1}^{\prime}\right)\right| \leq \frac{3}{2} \Lambda(r)\left|x_{2}^{\prime}-x_{1}^{\prime}\right| .
$$

Denoting $a=4 \Lambda(r) r$ and $y_{0}=(0,-a)$, we claim that $B_{r-a}\left(y_{0}\right) \cap \Omega$ is star-shaped with respect to $x_{0}$. Indeed, if $\left(x_{1}^{\prime}, \phi\left(x_{1}^{\prime}\right)\right),\left(x_{2}^{\prime}, \phi\left(x_{2}^{\prime}\right)\right) \in B_{1}$ are arbitrary points for which $x_{1}^{\prime} \neq x_{2}^{\prime}$ and $x_{2}^{\prime} \neq 0$, then

$$
\frac{\left|\phi\left(x_{2}^{\prime}\right)+a\right|}{\left|x_{2}^{\prime}\right|} \geq \frac{a-\left|\phi\left(x_{2}^{\prime}\right)\right|}{\left|x_{2}^{\prime}\right|} \geq \frac{4 \Lambda(r) r}{\left|x_{2}^{\prime}\right|}-\frac{3}{2} \Lambda(r) \geq \frac{5}{3} \Lambda(r)>\frac{3}{2} \Lambda(r) \geq \frac{\left|\phi\left(x_{2}^{\prime}\right)-\phi\left(x_{1}^{\prime}\right)\right|}{\left|x_{2}^{\prime}-x_{1}^{\prime}\right|},
$$

and thus the points $(0,-a),\left(x_{1}^{\prime}, \phi\left(x_{1}^{\prime}\right)\right)$, and $\left(x_{2}^{\prime}, \phi\left(x_{2}^{\prime}\right)\right)$ are not collinear.

Next, we claim

$$
\log \frac{H(r / 2)}{H(r / 4)} \leq \frac{\log \frac{2+16 \Lambda(r)}{1-16 \Lambda(r)}}{\log \frac{4-16 \Lambda(r)}{2+16 \Lambda(r)}} \log \frac{H(r)}{H(r / 2)} .
$$

For this purpose, let $r_{1}=r / 4-a, r_{2}=r / 2+a$, and $r_{3}=r-a$. Note that, since $\Lambda(r)<1 / 16$, we have $0<r_{1}<r_{2}<r_{3}<0$. Lemma 3.1 then implies

$$
\log \frac{H_{y_{0}}\left(r_{2}\right)}{H_{y_{0}}\left(r_{1}\right)} \leq \frac{\log \frac{r / 2+a}{r / 4-a}}{\log \frac{r-a}{r / 2+a}} \log \frac{H_{y_{0}}\left(r_{3}\right)}{H_{y_{0}}\left(r_{2}\right)} .
$$

Now, noting that $B_{r_{1}}\left(y_{0}\right) \subseteq B_{r / 4}, B_{r_{2}}\left(y_{0}\right) \supseteq B_{r / 2}$, and $B_{r_{3}}\left(y_{0}\right) \subseteq B_{r}$, we get (3.7).

By using (3.7) for $r=2^{-m}$ with $m=0,1, \ldots$ and then iterating the obtained inequalities, we get

$$
H\left(2^{-m}\right) \leq\left(\frac{H(1)}{H(1 / 2)}\right)^{C_{1}} H\left(2^{-m-1}\right), \quad m=1,2, \ldots,
$$

and Lemma 3.2 follows. 
Proof of Theorem 2.2. Note that, in the setting of Lemma 3.2,

$$
\sup _{x_{0} \in B_{1} \cap \partial \Omega} \frac{H_{x_{0}}(1)}{H_{x_{0}}(1 / 2)}<\infty
$$

and Theorem 2.2 follows from Lemma 3.2 .

\section{ACKNOWLEDGMENTS}

The work was partially supported by the NSF Grant DMS-9623161, by the MZT Slovenia Grant J1-7037-0101-95, and by a postdoctoral fellowship from the Swedish Research Council for Natural Sciences.

\section{REFERENCES}

[A] F. J. Almgren, Jr., Dirichlet's problem for multiple valued functions and the regularity of mass minimizing integral currents, Minimal Submanifolds and Geodesics, North-Holland, Amsterdam, M. Obata, 1979, pp. 1-6. MR 82g:49038

[AE] V. Adolfsson and L. Escauriaza, $C^{1, \alpha}$ domains and unique continuation at the boundary, 1996.

[AEK] V. Adolfsson, L. Escauriaza, and C. Kenig, Convex domains and unique continuation at the boundary, Revista Matemática Iberoamericana 11 (1995), 513-525. MR 96j:31003

[K] I. Kukavica, Level sets for the stationary Ginzburg-Landau equation, 1996, to appear in Calc. Var. PDE.

[L] F.-H. Lin, Nodal sets of solutions of elliptic and parabolic equations, Comm. Pure Appl. Math. 44 (1991), 287-308. MR 92b:58224

Department of Mathematics, The University of Chicago, Chicago, Illinois 60637

E-mail address: kukavica@cs.uchicago.edu

Current address: Department of Mathematics, University of Southern California, Los Angeles, California 90089

E-mail address: kaj@math.uchicago.edu 